\title{
The Prevalence and Predictors of Non-alcoholic Fatty Liver Disease in Morbidly Obese Women - A Cross-sectional Study from Southern India
}

\author{
Avica Atri, ${ }^{1}$ Stephen A Jiwanmall, ${ }^{2}$ Munaf B Nandyal, ${ }^{2}$ Dheeraj Kattula, ${ }^{2}$ Sandhiya Paravathareddy, ${ }^{1}$ Thomas V Paul, \\ Nihal Thomas ${ }^{1}$ and Nitin Kapoor ${ }^{1,3}$ \\ 1. Department of Endocrinology, Diabetes and Metabolism, Christian Medical College and Hospital, Vellore, Tamil Nadu, India: \\ 2. Department of Psychiatry, Christian Medical College and Hospital, Vellore, Tamil Nadu, India; 3. Noncommunicable Disease Unit, \\ Melbourne School of Population and Global Health, University of Melbourne, Victoria, Australia
}

DOl: https://doi.org/10.17925/EE.2020.16.2.152

$\mathrm{B}$ ackground: The prevalence of obesity is increasing rapidly in India and so are its associated comorbidities. Non-alcoholic fatty liver disease (NAFLD), the hepatic manifestation of metabolic syndrome, is commonly associated with obesity. However, limited data are available on its prevalence and clinical indicators among morbidly obese Indian women. The aim of our study was to find the prevalence of NAFLD in morbidly obese Indian women and study the clinically measurable obesity indicators that would best predict NAFLD. Methods: This was a cross-sectional study, conducted in the Department of Endocrinology, Diabetes and Metabolism, Christian Medical College, Vellore. Women were enrolled who were diagnosed to have NAFLD on sonography. Anthropometric variables, such as body mass index, waist circumference, hip circumference, waist-hip ratio and waist-height ratio were measured and compared between the two groups. SPSS Statistics 21.0 software was used for analysing the data. Results: One hundred and six consecutive, morbidly obese women were recruited in this study. Nearly three-quarters (73.6\%) of the 106 morbidly obese participants were found to have NAFLD. We found waist circumference, body mass index and waist-height ratio to be most useful in distinguishing between patients with and without NAFLD, and found waist-height ratio was the best screening tool for diagnosing NAFLD. Conclusion: NAFLD is present in a large proportion of morbidly obese women. Waist-height ratio could be used a surrogate screening tool to detect NAFLD in resource-constrained settings.

\section{Keywords}

Anthropometry, morbidly obese, non-alcoholic fatty liver disease (NAFLD), obesity indices, waist circumference, waist-height ratio

Disclosures: Avica Atri, Stephen A Jiwanmall, Munaf B Nandyal, Dheeraj Kattula, Sandhiya Paravathareddy, Thomas V Paul, Nihal Thomas and Nitin Kapoor have no financial or non-financial relationships or activities to declare in relation to this article.

\section{Review Process: Double-blind peer review.}

Compliance with Ethics: All procedures were followed in accordance with the responsible committee on human experimentation and with the Helsinki Declaration of 1975 and subsequent revisions. Approval was granted by the Institutional Research Board - minute number 10146 (22.06.2016). Informed consent was obtained from the patients involved in this study.

Authorship: All named authors meet the criteria of the International Committee of Medical Journal Editors (ICMJE) for authorship for this manuscript, take responsibility for the integrity of the work as a whole and have given final approval for the version to be published.

Access: This article is freely accessible at

touchENDOCRINOLOGY.com

(C) Touch Medical Media 2020

Received: 15 April 2020

Accepted: 15 June 2020

Published Online: 6 October 2020

Citation: European Endocrinology. 2020;16(2):152-5

Corresponding Author: Nitin Kapoor, Department of Endocrinology, Diabetes and Metabolism, Christian Medical College, Vellore, Tamil Nadu 632004, India.

E: nitin.endocrine@gmail.com

Support: No funding was received in

the publication of this article.
The prevalence of obesity is rapidly increasing across the world. In India, about $12.6 \%$ women and 9.3\% men are obese, amounting to more than a 100 million population. ${ }^{1}$ Obesity, which was earlier considered to be a growing problem only in metropolitan cities, is now noted to be just as prevalent in smaller semi-urban towns. ${ }^{2}$ It is not an imminently fatal disease in itself, but is associated with a wide range of serious non-communicable disorders, such as metabolic syndrome, hypertension, atherosclerosis, heart disease, diabetes, dyslipidaemia and sleep disorders. Non-alcoholic fatty liver disease (NAFLD), the hepatic manifestation of the metabolic syndrome, is most commonly associated with obesity. ${ }^{3}$ Among Indians, obesity is more commonly seen in women compared to men and is increasing in prevalence in children and adolescents, due to a sedentary lifestyle and unhealthy eating, along with which a genetic component has also been hypothesised. ${ }^{4-7}$

The development of these metabolic derangements is not only related to excess energy intake, but more importantly, to an altered lipid metabolism. Ectopic fat accumulation (in non-adipose tissue like the skeletal muscle, the pancreas, the liver and intra-abdominally) appears to be a key feature in distinguishing those who develop metabolic derangements from those who will not. ${ }^{8}$ In this way, fat accumulation in the liver due to non-alcoholic causes (NAFLD) is a robust, independent marker of metabolic dysfunction, which occurs due to increased fatty acid transfer away from, rather than into, the adipose tissue, and has therefore been considered the major problem in the progression from health to disease. ${ }^{8}$ One theory explaining this is the 'lipid overflow hypothesis', proposed by Roger Unger in 2003,' according to whom, the metabolic alterations associated with obesity result from leptin resistance and a failure in the capacity for appropriate adipose tissue expansion with consequent storage of surplus lipids in non-adipose tissues, resulting in lipotoxicity.

People of Asian origin tend to have a higher proportion of body fat than whites, and more of it is distributed within the abdominal cavity as visceral adipose tissue. ${ }^{10}$ The distinctive features of obesity in Asian Indians include greater body fat percentage and truncal, intra-abdominal and subcutaneous adipose tissue compared to whites, and are more insulin resistant than whites, probably due to the increased visceral fat, and are therefore more prone to obesity and the complications of the same. ${ }^{4,10,11}$ Moreover, Asian Indians are more prone to insulin resistance and 
have increased waist circumference and body fat (particularly visceral fat), features that are described as the Asian Indian phenotype. 12,13 Asians have higher weight-related disease risks at lower body mass indexes (BMIS), the possible explanation for which, is body fat distribution. When compared to white Europeans with a similar BMI, Asians have a 3-5\% higher total body fat. ${ }^{14}$ The cause of this racial and ethnic disparity is most likely multifactorial and includes contributions from genetic, behavioural and socioeconomic factors.

Based on the World Health Organization (WHO) guidelines for BMI, a diagnosis of overweight and obese was made, with cut-offs of $\geq 23 \mathrm{~kg} / \mathrm{m}^{2}$ and $\geq 25 \mathrm{~kg} / \mathrm{m}^{2}$, respectively, as per the Consensus Guidelines for Asian Indians. ${ }^{15}$ In addition to BMI, there are various other anthropometric indices that can be clinically measured, and may be more accurate markers of obesity-related comorbidities. Some of these are waist circumference, hip circumference, waist-hip ratio and waist-height ratio. ${ }^{16}$ However, the utility of these indicators in the morbidly obese is unknown.

NAFLD refers to the presence of hepatic steatosis with or without fibrosis, when no other causes for secondary hepatic fat accumulation are present. ${ }^{3}$ Clinically, it ranges from simple steatosis, hepatitis, fibrosis, cirrhosis, and rarely hepatocellular carcinoma. The prevalence of NAFLD in Western countries has been found to be $20-30 \%$ in the general population, and about $80-90 \%$ among obese adults. ${ }^{17}$ In India, it is said to be around $9-32 \%$ in the general population, with a higher incidence rate amongst people with obesity and people with diabetes. ${ }^{6}$ The highest prevalence of NAFLD has been reported from South America and the Middle East, whereas the lowest rate was reported from Africa. ${ }^{18}$

Obesity (excessive BMI and visceral obesity) is the most common and well documented risk factor for NAFLD. ${ }^{19}$ Indeed, the entire spectrum of obesity, ranging from overweight to obese and severely obese, is associated with NAFLD. There are limited data on the prevalence of NAFLD from India and on the clinical predictors of NAFLD among patients who are morbidly obese. Therefore, we have correlated some of these non-conventional obesity indices with the Edmonton Obesity Staging System (EOSS; a five-stage system of obesity that provides a score from 0-4 after considering the metabolic, physical, and psychological parameters), to find the best clinical predictor of NAFLD in morbidly obese subjects, as BMI alone has not been seen to be a satisfactory indicator. ${ }^{20-22}$ Also, it may not be feasible to do ultrasound scans for all patients who are morbidly obese due to a lack of facilities and patient financial constraints; therefore, finding good clinical indices would be practically relevant.

The aim of this study was to determine the prevalence of NAFLD and the best clinical correlate of NAFLD including the most appropriate cut-off limit, using non-conventional obesity indices, in women who are morbidly obese. This may serve to be a better predictor than BMI alone, for use in clinical practice.

\section{Patients and methods}

This study was a cross-sectional study conducted in the Department of Endocrinology, Christian Medical College, Vellore, between 1 November 2017 and 30 October 2018. Approval was granted by the Institutional Research Board - minute number 10146 (22.06.2016).

Among Indians, complications of obesity can occur at a lower BMI than that of non-Indians. ${ }^{12,23}$ Patients with a $\mathrm{BMl}>32.5 \mathrm{~kg} / \mathrm{m}^{2}$ and comorbidities are often eligible for bariatric surgery; therefore, women with a BMI of $\geq 32.5 \mathrm{~kg} / \mathrm{m}^{2}$ (morbidly obese) who visited the bariatric clinic and had an abdominal ultrasound for a non-hepatic indication, were consecutively included in the study. ${ }^{24}$ Exclusion criteria included patients who had a significant alcohol consumption (current or recent alcohol consumption of $>20 \mathrm{~g} /$ day in women) and patients with other causes of chronic liver disease (such as hepatitis or family history of congenital liver disease). Abdominal ultrasound was used as the reference for diagnosing NAFLD among the study population. All ultrasound scans used in this study to diagnose NAFLD were done in the radiology department in the same hospital, thus minimising any possible variability in reports. Obesity indicators were measured using the standard WHO STEPwise approach to surveillance (STEPS) protocols. ${ }^{25}$

Waist circumference was measured at the approximate midpoint between the lower margin of the last palpable rib and the top of the iliac crest, along the mid axillary line, at the end of a gentle exhalation. Hip circumference was measured at the level of the widest circumference over the buttocks. Both of these parameters were measured in standing subjects, with arms by their side, using a static tape, with the tape parallel to the ground, being loose enough to allow the observer to place one finger between the tape and the subject's body. Height was measured with a wall-mounted stadiometer to the nearest $0.1 \mathrm{~cm}$. Weight was measured in kilograms (to the nearest $100 \mathrm{~g}$ ) using an electronic scale. All readings were taken with the subject having no extra layers of clothing or full pockets, after removing their shoes and without other heavy objects on them, such as any heavy jewellery and/or watches. Measurements were recorded by an observer who was seated in front of the patient; the same trained observer who had been working in the clinic since its inception was assigned to measure the anthropometric indices for all patients, to minimise interobserver bias.

For every patient, based on their clinical features, psychiatric assessment and laboratory information obtained, we also calculated an EOSS score to correlate with the presence or absence of NAFLD. After collecting clinical and anthropometric data, participants were divided into two groups based on whether they had NAFLD or not (based on abdominal ultrasound). Following this, their data were compared and results were obtained.

SPSS Statistics 21.0 software was used for analysing the data. For all variables with a normal distribution, the mean and standard deviation (SD) was obtained. Area under the curve (AUC) and (receiver operating characteristic) ROC curves were used to identify the variables to be used as screening tools. Continuous variables were presented as mean \pm SD or median with range, depending on the distribution of the variable. Categorical variables were expressed as frequencies or percentages. Sensitivity, specificity and ROC curves for each anthropometric variable of interest in prediction of NAFLD, were estimated. The Strengthening the Reporting of Observational studies in Epidemiology (STROBE) reporting guidelines were used to draft the manuscript. ${ }^{26}$

\section{Results}

A total of 106 women were included in this study. Mean ( \pm SD) age of the participants was $40.2 \pm 11$ years, and the mean BMI $( \pm$ SD) was $41.58 \pm 5.8 \mathrm{~kg} / \mathrm{m}^{2}$. The majority of the patients were from Tamil Nadu (59.4\%) and West Bengal (12.3\%). The prevalence of NAFLD was found to be $73.6 \%$ (95\% confidence interval $[\mathrm{Cl}]$ 65.2-82.0). After comparing the various non-conventional anthropometric indices between those with and without NAFLD, there were significant differences in their BMI and waist-height ratio ( $p$-values $<0.05$ ); whereas weight, height, waist circumference, hip circumference and waist-hip ratio were not found to be individually useful as clinical predictors of NAFLD, as seen in Table 1. 
Table 1: Comparison of anthropometric indices between those with and without NAFLD

\begin{tabular}{|c|c|c|c|c|}
\hline & $\begin{array}{l}\text { Total } \\
(n=106)\end{array}$ & $\begin{array}{l}\text { With NAFLD } \\
(\mathrm{n}=78)\end{array}$ & $\begin{array}{l}\text { Without NAFLD } \\
(\mathrm{n}=28)\end{array}$ & $p$ value \\
\hline Age, years & $40.2(11)$ & $40.9(11)$ & $38.4(10.8)$ & 0.296 \\
\hline Height, cm & $155.7(6.7)$ & $155.2(6.3)$ & $157.1(7.6)$ & 0.228 \\
\hline Weight, kg & $101.3(16.8)$ & $102.2(16.7)$ & $99.0(17.1)$ & 0.406 \\
\hline $\mathrm{BMI}, \mathrm{kg} / \mathrm{m}^{2}$ & $41.6(5.8)$ & $42.3(6.1)$ & $39.7(4.4)$ & $0.021^{*}$ \\
\hline Waist circumference, $\mathrm{cm}$ & $117.7(14.2)$ & $120.0(14.1)$ & $111.3(12.7)$ & $0.004^{*}$ \\
\hline Hip circumference, cm & $126.1(13.5)$ & $126.8(13.3)$ & $124.0(13.8)$ & 0.358 \\
\hline WH ratio & $0.935(0.1)$ & $0.945(0.1)$ & $0.906(0.1)$ & 0.108 \\
\hline WHt ratio & $0.757(0.1)$ & $0.775(0.1)$ & $0.709(0.1)$ & $0.001^{*}$ \\
\hline
\end{tabular}

All values are reported as mean (SD). *Indices with statistically significant differences, $p<0.05$.

$B M I=$ body mass index; NAFLD = non-alcoholic fatty liver disease; $S D=$ standard deviation; $W H$ = waist-hip; WHt = waist-height.

Table 2: Results of ROC analysis between the various anthropometric indices

\begin{tabular}{|l|l|l|l|}
\hline & AUROC & p value & $\begin{array}{l}95 \% \text { confidence } \\
\text { interval }\end{array}$ \\
\hline WHt ratio & 0.714 & 0.001 & $0.598-0.831^{*}$ \\
\hline Waist circumference & 0.691 & 0.003 & $0.572-0.810^{*}$ \\
\hline WH ratio & 0.632 & 0.042 & $0.503-0.760 *$ \\
\hline BMI & 0.616 & 0.069 & $0.500-0.733$ \\
\hline Height & 0.570 & 0.276 & $0.444-0.695$ \\
\hline Hip circumference & 0.537 & 0.571 & $0.413-0.661$ \\
\hline Weight & 0.430 & 0.271 & $0.300-0.560$ \\
\hline
\end{tabular}

*Indices with statistically significant differences, $p<0.05$.

$A U R O C=$ area under the ROC curve; $B M I=$ body mass index; $R O C=$ receiver operating characteristic; $W H=$ waist-hip; $W H t=$ waist-height.

Comparing comorbidities, such as diabetes, hypertension, osteoarthritis, obstructive sleep apnoea, polycystic ovary syndrome, gastroesophageal reflux disease and hernias, there was no statistically significant difference seen in their prevalence between those with NAFLD and without. Additionally, presence of these comorbidities could not predict a higher possibility of NAFLD among an obese population; although comorbidities were more prevalent among the group with NAFLD. Of the patients included, 72/106 (67.9\%) had hyperglycaemia (diabetes mellitus/impaired glucose tolerance), 49/106 (46.2\%) had hypertension, 28/106 (26.4\%) had osteoarthritis, 50/106 (47.2\%) had dyslipidaemia, 28/106 (26.4\%) had suspected obstructive sleep apnoea, 18/106 (17.0\%) had polycystic ovary syndrome and 14/106 (13.2\%) had gastroesophageal reflux. Participants were also scored based on the EOSS, and it was seen that $88 \%$ of those with NAFLD, had an EOSS score $\geq 2$, compared with $71 \%$ of those without NAFLD, having a mean difference of $17 \%$ which was significantly different $(p=0.039)$.

We plotted ROC curves to find the best anthropometric variable that could be used as a screening tool for NAFLD in a cohort of morbidly obese women. Waist-height ratio was found to be a somewhat better screening tool (AUC 0.714) in women who are morbidly obese (Table 2) to identify a subset with NAFLD, with a cut off of 0.700 , that would have a sensitivity of $73 \%$ and specificity of $63.6 \%$.

\section{Discussion}

Nearly three-quarters (73.6\%) of the 106 morbidly obese women included in this study had NAFLD. Among the various clinical anthropometric indices considered, waist circumference, BMI and waist-height ratio were noted to be most useful in distinguishing between patients with and without NAFLD. But the best clinical predictor that can also be used as a screening tool for NALFD, based on the ROC analysis among these patients, was concluded to be waist-height ratio. We also found no difference in the presence of various other comorbidities between women with and without NAFLD. On studying the EOSS scores of all the patients included in the study, a larger proportion of the women with NAFLD had an EOSS $\geq 2$, compared with those without.

The estimated prevalence of NAFLD in the general population, as seen in a study by Clark, ranges from 3-24\%, with most estimates in the 6-14\% range, and it is more common among patients undergoing bariatric surgery, ranging from $84-96 \% .{ }^{27}$ In India, the prevalence of NAFLD is found to be around $10-32 \%$ in the general population, but as expected the prevalence in an obese population is much higher. ${ }^{28}$ In our study, the prevalence of NAFLD was found to be $73.6 \%$, with a mean BMI of $41.58 \pm 5.8 \mathrm{~kg} / \mathrm{m}^{2}$, using ultrasound scans for diagnosis of NAFLD; whereas, in a study among 143 South Indian patients who were morbidly obese undergoing bariatric surgery, the mean BMl was $44.66 \pm 9.81 \mathrm{~kg} / \mathrm{m}^{2}$, and the prevalence of NAFLD based on histological diagnosis by liver biopsy was, as expected, slightly lower than ours, at $65.7 \%(n=88){ }^{29}$

In a study conducted in Bangladesh on 493 patients with sonographic evidence of fatty hepatic alteration, $75 \%$ of whom were obese, it was seen that the presence of diabetes could significantly $(p=0.001)$ differentiate non-alcoholic steatohepatitis from simple steatosis, while age, waist circumference, triglyceride, insulin resistance index and hypertension could not. ${ }^{30}$ In a study conducted among young adults in South India to analyse the relationship between various anthropometric indices and metabolic syndrome, waist circumference and skin-fold thickness were found to be significantly associated with metabolic abnormalities. ${ }^{31}$ Unlike others, in our study, BMI, waist circumference and waist-height ratio were noted to be good anthropometric predictors of NAFLD, with no relation between comorbidities like diabetes mellitus and hypertension and the presence of NAFLD.

A study from Israel on the risk factors and predictive anthropometric indices of NAFLD, found that abdominal obesity was independently associated with NAFLD ( $p<0.05$ for BMl and waist circumference). ${ }^{32}$ A similar study in an Indian population in 60 patients who were obese with a $\mathrm{BMI} \geq 30\left(36.15 \pm 4.20 \mathrm{~kg} / \mathrm{m}^{2}\right)$, in whom NAFLD was diagnosed by ultrasound scan, showed the prevalence of NAFLD to be $80 \%$, with a significant difference between BMI and waist-hip ratio between the two groups. ${ }^{33}$ In a review looking at Western populations, the presence 
of multiple metabolic disorders was found to be associated with a potentially progressive severe liver disease, alongside increasing prevalence of obesity, coupled with diabetes, dyslipidaemia, hypertension and ultimately metabolic syndrome. ${ }^{34}$ This puts a very large population at risk of forthcoming liver failure in the next few decades..$^{13,33-35}$

This is the first study undertaken in India which looks at anthropometric indices other than BMI as predictors of NAFLD, in a specific cohort of women who are morbidly obese. It thus caters to the lack of adequate data on clinical predictors of NAFLD among patients who are morbidly obese, and the association with various risk factors. In this way, the need for unnecessary ultrasound scans in clinical practice in all patients who are morbidly obese to rule out NAFLD can be reduced, by only doing ultrasound scans in a more focused subgroup of those who are morbidly obese after using waist-height ratio as a screening tool and taking into consideration the patient's waist circumference and BMI and waist-height ratio. The clinical utility of such anthropometric measurements increases drastically in low-middle income countries like India, where not all primary healthcare setups have access to ultrasound facilities and patient financial constraints are often a big burden.

One of the limitations of this study is that although the gold standard for diagnosing NAFLD is liver biopsy, we could not use this for diagnosis, as it was not practically possible. Also, it is possible that the intra-abdominal visibility in an ultrasound scan can be diminished in patients who are obese due to subcutaneous adipose tissue. Since this study has been conducted in a tertiary care centre, there may be a reference bias. The cross-sectional, observational design of the study and the small sample size are other drawbacks of the study, and a prospective, randomised, controlled study in larger number of patients in the future is warranted to further highlight the predictors of NAFLD, while considering the differences between the radiological methods, such as ultrasound, computed tomography scan, magnetic resonance imaging, and the histopathological diagnosis of NAFLD. As it was conducted among females, its applicability to males may need to be further studied. In addition, as this study was done in a hospital setting, further studies in the community may be required for application of these results to the population at large.

Through this study we learnt that the most relevant anthropometric indices to predict NAFLD in a cohort of women who are morbidly obese are BMI, waist circumference and waist-height ratio, and BMI alone does not provide an accurate predictive parameter. We also identified an appropriate cut-off for waist-height ratio that can be used as a screening tool among women who are morbidly obese, although more studies may be required to apply it to the population at large. $\square$
1. Ahirwar R, Mondal PR. Prevalence of obesity in India: $A$ systematic review. Diabetes Metab Syndr. 2019;13:318-21.

2. Ranjani H, Mehreen TS, Pradeepa R, et al. Epidemiology of childhood overweight \& obesity in India: A systematic review. Indian J Med Res. 2016;143:160-74.

3. Byrne CD, Targher G. NAFLD: a multisystem disease. J Hepatol. 2015;62(Suppl. 1):S47-64.

4. Misra A, Shrivastava U. Obesity and dyslipidemia in South Asians. Nutrients. 2013;5:2708-33.

5. Baxi R, Vasan SK, Hansdak S, et al. Parental determinants of metabolic syndrome among adolescent Asian Indians: cross-sectional analysis of parent-offspring trios. J Diabetes. cross-sectional anal.

6. Misra A, Singhal N, Khurana L. Obesity, the metabolic syndrome, and type 2 diabetes in developing countries: role of dietary fats and oils. J Am Coll Nutr. 2010;29(Suppl. 3):289-301S

7. Vasan SK, Fall T, Neville MJ, et al. Associations of variants in FTO and near MC4R with obesity traits in South Asian Indians. Obesity. 2012;20:2268-77.

8. Mittendorfer B. Origins of metabolic complications in obesity adipose tissue and free fatty acid trafficking. Curr Opin Clin Nutr Metab Care. 2011;14:535-41.

9. Unger RH. Lipid overload and overflow: metabolic trauma and the metabolic syndrome. Trends Endocrinol Metab. 2003;14:398-403.

10. Raji A, Seely EW, Arky RA, Simonson DC. Body fat distribution and insulin resistance in healthy Asian Indians and Caucasians. and insulin resistance in healthy Asian Ind

11. Shelgikar KM, Yajnik CS, Hockaday TDR. Central rather than generalized obesity is related to hyperglycaemia in Asian Indian subjects. Diabetic Medicine. 1991;8:712-17.

12. Kapoor N, Furler J, Paul TV, et al. Ethnicity-specific cut-offs that predict co-morbidities: the way forward for optimal utility of obesity indicators. J Biosoc Sci. 2019;51:624-6.

13. Kapoor N, Furler J, Paul TV, et al. Normal weight obesity: an underrecognized problem in individuals of South Asian descent. Clin Ther. 2019;41:1638-42.

14. WHO Expert Consultation. Appropriate body-mass index for Asian populations and its implications for policy and intervention strategies. Lancet. 2004;363:157-63.

15. Misra A, Chowbey P, Makkar BM, et al. Consensus statement for diagnosis of obesity, abdominal obesity and the metabolic syndrome for Asian Indians and recommendations for physica activity, medical and surgical management. J Assoc Physicians India. 2009:57:163-70.

16. Kapoor $\mathrm{N}$, Lotfaliany $\mathrm{M}$, Sathish $\mathrm{T}$, et al. Obesity indicators that best predict type 2 diabetes in an Indian population: insights from the Kerala Diabetes Prevention Program. J Nutr Sci. from the Ker

17. Bellentani $\mathrm{S}$. The epidemiology of non-alcoholic fatty liver disease. Liver Int. 2017;37(Suppl. 1):81-4.

18. Pan JJ, Fallon MB. Gender and racial differences in nonalcoholic fatty liver disease. World I Hepatol. 2014;6:274-83.

19. Marchesini G, Moscatiello S, Di Domizio S, Forlani G Obesity-associated liver disease. I Clin Endocrinol Metab. 2008;93(Suppl. 1):S74-80.

20. Canning KL, Brown RE, Wharton S, et al. Edmonton obesity staging system prevalence and association with weight loss in a publicly funded referral-based obesity clinic. J Obes. 2015;2015:619734.

21. Jiwanmall $S A$, Kattula $D$, Nandyal MB, et al. Psychiatric burden in the morbidly obese in multidisciplinary bariatric clinic in South the morbidly obese in multidisciplinary bariatic

22. Sharma AM, Kushner RF. A proposed clinical staging system for Sharma AM, Kushner RF. A proposed clinica
obesity. Int J Obes (Lond). 2009:33:289-95.

23. Kapoor N, Furler J, Paul TV, et al. The BMI-adiposity conundrum in South Asian populations: need for further research. J Biosoc Sci. 2019;:51:619-21.

24. Behl S, Misra A. Management of obesity in adult Asian Indians Indian Heart J. 2017;69:539-44.

25. World Health Organization. WHO STEPS Surveillance Manual: The WHO STEPwise approach to chronic disease risk factor surveillance. 2005. Available at: https://apps.who. int/iris/bitstream/handle/10665/43376/9241593830 eng. pdf? sequence=1\&isAllowed=y (accessed 12 August 2020).

26. Vandenbroucke JP, von Elm E, Altman DG, et al. Strengthening the Reporting of Observational Studies in Epidemiology (STROBE): explanation and elaboration. Int I Surg. 2014;12:1500-24.

27. Clark JM. The epidemiology of nonalcoholic fatty liver disease in adults. J Clin Gastroenterol. 2006;40(Suppl. 1):S5-10.

28. Singh SP, Panigrahi MK. Guidelines for assessment and management of nonalcoholic fatty liver disease in Indians. 2013. Available at: $\mathrm{http}$ ://scbmch.nic.in/pdf/Guidelines\%20 for\%20Assessment\%20\%\%20Mgt\%20of\%20NAFLD\%20in\%20 for\%20Assessment\%20\&\%20Mgt\%200\%20NAFLD).
Indians\%20-\%202013.pdf (accessed 19 June 2020).

Indians\%20-\%202013.pdf (accessed 19 June 2020).
29. Praveenraj P, Gomes RM, Kumar S, et al. Prevalence and predictors of non-alcoholic fatty liver disease in morbidly obese South Indian patients undergoing bariatric surgery. Obes Surg. 2015;25:2078-87.

30. Alam S, Noor-E-Alam SM, Chowdhury ZR, et al. Nonalcoholic steatohepatitis in nonalcoholic fatty liver disease patients of Bangladesh. World J Hepatol. 2013;5:281-7.

31. Vasan SK, Thomas N, Christopher S, et al. Anthropometric measurements for the prediction of the metabolic syndrome: a cross-sectional study on adolescents and young adults from Southern India. Heart Asia. 2011;3:2-7.

32. Zelber-Sagi S, Nitzan-Kaluski D, Halpern Z, Oren R. Prevalence of primary non-alcoholic fatty liver disease in a population-based study and its association with biochemical and anthropometric study and its association with biochemic

33. Patell R, Dosi R, Joshi H, et al. Non-alcoholic fatty liver disease (NAFLD) in obesity. J Clin Diagn Res. 2014;8:62-6.

34. Benedict M, Zhang X. Non-alcoholic fatty liver disease: an expanded review. World J Hepatol. 2017;9:715-32

35. Kapoor N, Audsley J, Rupali P, et al. A gathering storm: HIV infection and nonalcoholic fatty liver disease in low and middle-income countries. AIDS. 2019:33:1105-15. 